Widefield Science and Technology for the SKA

SKADS CONFERENCE 2009

S.A. Torchinsky, A. van Ardenne, T. van den Brink-Havinga, A.J.J. van Es, A.J. Faulkner (eds.)

4-6 November 2009, Château de Limelette, Belgium

\title{
Aperture Array Frontend Design for Production
}

\author{
M. Drost, R.H. van den Brink, H.H. Hanenburg, and J.H. Pragt
}

\begin{abstract}
ASTRON, Netherlands Institute for Radio Astronomy, The Netherlands
\end{abstract}
\begin{abstract}
Aperture Arrays (AA) for astronomical telescopes are special large size, dense structured, broadband and three dimensional shaped radio sensor systems. The AA systems for astronomical applications development, in particular SKA, starts at the end of the $20^{\text {th }}$ century at ASTRON with system demonstrators at TRL (Technology Readiness Level) level 2-3 (Research to Prove Feasibility) with sizes of one square meter. These successful system demonstrators are succeeded by projects with a continuous increase of focus into mechanical design and in special the design for production and assembly towards low-cost. Recent projects like EMBRACE show a continuous reduction of material, production and assembly cost with an improvement in performance. This project deliverable will be on TRL level 6-7 (Technology demonstrator). In the same time an AA called LOFAR is developed from TRL level 3 towards the highest and final TRL level 8-9 (System Test, Launch and Operation). This low frequency $(<250 \mathrm{MHz})$ array shows that the front end of a broadband AA can be simple and relative low cost in combination with extreme large collecting area. Other AA developments are maturing using extreme low-cost materials like foam and foil. The one square meter system demonstrator FLOWPAD ${ }^{3}$ and six square meters 2PAD are at TRL level 5-6 and look promising for application into the $\mathrm{GHz}$ domain. The production techniques for low-cost AA constructions are also applied in the most recent Focal Plane Arrays designs like APERTIF and DIGESTIF.

This paper presents the design, construction, production and assembly of different Aperture Array telescopes, demonstrators and Focal Plane Arrays instruments with Technology Readiness Levels of 2 to 9.
\end{abstract}

\section{Introduction}

There are several reasons from a mechanical point of view to use an aperture array for the SKA. At first, due to the increased demand for collecting area, the traditional dish structures are getting larger and heavier. This results in more extreme strength and stiffness of instruments and their parts. The costs go up and the second build will cost just as much as the first.

When using an aperture array no moving parts are required, but still the ability exists for steering the beam. Even it is possible to use multi independent field of views at the same time decreasing the observing time. When the stiffness and strength of materials and parts are less demanding, more low cost materials can be used decreasing the overall cost. The general idea of using an aperture array is using a large number of small identical parts out of low cost materials, together forming a large instrument, instead of building several large expensive parts out of classical materials. The large number of identical small parts can be assembled with typical low cost consumer parts and produced by several different mass production processes. The large number of identical parts, modular design, mass production and consumer-market assembly processes are the key to large cost effective SKA Aperture Arrays.

The consumer market production processes are typically focused on reduction of nonproductive effort. This means an employee is instructed to do one task as part of a complete task list. This way the required tools are always at hand and less or no time is spent on retrieving or preparing materials. The time needed to manufacture a product using mass production is shorter than when using traditional methods. Also the probability of human error and variation is reduced, as tasks are predominantly carried out by machinery. A reduction in labour costs, as well as an increased rate of production, enables a com-

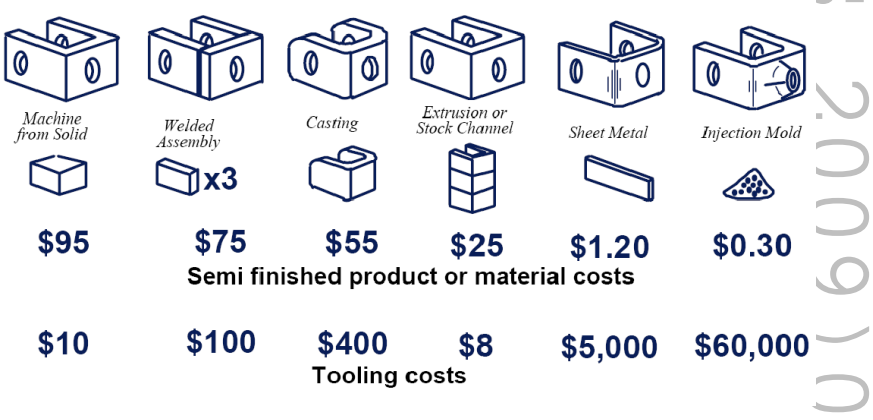

Fig. 1: recurring and non-recurring costPhilpott (2009)

pany to produce a larger quantity of one product at lower cost than using traditional, non-linear methods. With mass production the costs for expensive tools and molds can be spread over the large number of parts resulting in the fact that the cost will primarily be defined by material and process time. The major disadvantage of mass production is the inflexibility after a production line is implemented. A well organized preparation is required and the design should be completely finished when production is started.

So building the SKA by the design of an aperture array, reduction of cost can be achieved by mass production enabled by identical consumer market parts (simplicity), a large amount of these parts (mass), the use of less demanding materials and parts (low cost materials) and a reduction of observation time (multi beaming)

The aforementioned cost of mass produced products is mainly driven by the material cost and its process time: choosing a low cost material is crucial. One group which excels in this aspect are the thermoplastics (PE, PP, PS, PVC, etc). With 


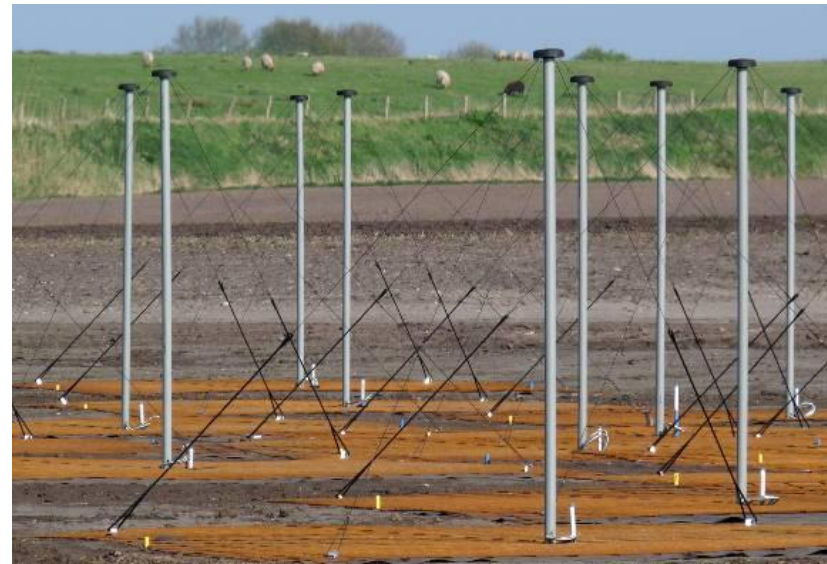

Fig. 2: LOFAR LBA

the selection of material, the availability of production processes is also narrowed. Again thermoplastics excel in this aspect, because one of the fastest production methods is injection molding. Another important feature of injection molding and thermoplastics is that the design freedom is enormous. Almost any product shape is possible to produce, which enables the integration of many functions which of course lowers the cost. Large drawback of these materials is that the mechanical properties like elasticity and strength are reduced compared to metals.

Fig. 1 shows why injection molding is only feasible for mass production purposes. The non-recurring cost, in this case the mold, is high and there for a large number of parts are necessary to smear out these costs. On the other side machining is used to realize this part. In this case material and process time are high and the non-recurring cost is low, no tooling is required. This is the main reason why machining is used for small production numbers.

\section{Cost reduction by simplicity}

Well known example of cost reduction by simplicity is the (Low Frequency Array) LOFAR front-end. The LOFAR frequency band is split into to a HBA and LBA sub band with each its own antenna design. The Low Band Array (LBA) is, from mechanical point of view, a good example of a simple antenna / front-end design. Where possible there is made use of COTS (Commercial off the Shelf) parts like the polyvinylchloride centre pole, the electric dipole wires, rope, tent pegs and concrete reinforcement-steel wire mesh. Advantage of this kind of design is that the parts are produced using a small subset of the available mass production capabilities, without any investment. The EPDM (rubber) springs are produced by rubber press shaping. The shape is reproduced by a mold in which the pressed elastic rubber is vulcanized. This production method is suitable for series of 1000 parts or more.

The two pieced mold is simple and low cost, no automatic filling and handling is required. The plastic rubber is placed by hand in the mold and heated and pressed such that the plastic

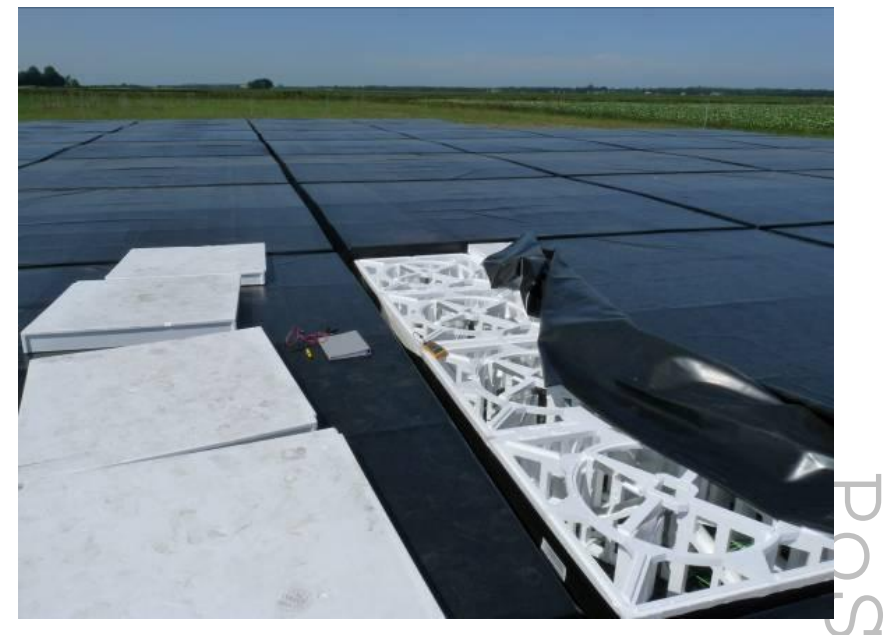

Fig. 3: LOFAR HBA tiles

rubber vulcanizes in the mold, with as result the elastic rubber spring.

The High Band Array (HBA) consists of a larger amount of identical parts then the LBA, making a more modular type of approach possible. The less demanding strength and stiffness of the construction enables mass production materials and processes. The construction is made of EPS, a material that is generally used for packaging and insulation purposes. The base material (PS) polystyrene is a low cost thermoplastic which is expanded, resulting in a low density $\left(25 \mathrm{~kg} / \mathrm{m}^{3}\right)$ and low cost foam.

Less material also means less strength and stiffness, still the structure needs to cope with people walking over the tiles and snow load. This is solved by applying a relative thick cover, which results in an acceptable stiffness and strength. The stiffness and strength are non-linear affected by the thickness. stiffness is raced to the third power and the strength goes up quadratic. The $10 \mathrm{~cm}$ thick EPS cover of the HBA is able to endure these loads, and has got the weight equivalent of 2.5 $\mathrm{mm}$ thick solid EPS. The $2.5 \mathrm{~mm}$ thick PS plate however can not withstand the described loads.

Due to the low weight of EPS it becomes possible to realize one $5 \times 5$ meter structure / module which has got a number of positive aspects: antenna plus electronics are protected from the environment, assembly and testing can be done within indoor conditions, which increases the reliability significantly, installation time in the field under varying weather conditions is shortened and maintenance cost is lowered because the HBA array covers a large ground area so maintaining the vegetation is limited to a minimum.

The antenna elements are stamped out of aluminum sheet metal, which is an automated process suitable for large series. The antenna elements are then assembled by clicking them into the EPS structure. The whole $5 \times 5$ meter tile is covered by a FPP bottom and top bag, which is firmly anchored into the ground by a COTS anchor system.

Both designs, Low Band Array and High Band Array, were developed towards the highest and final level of the NASA 


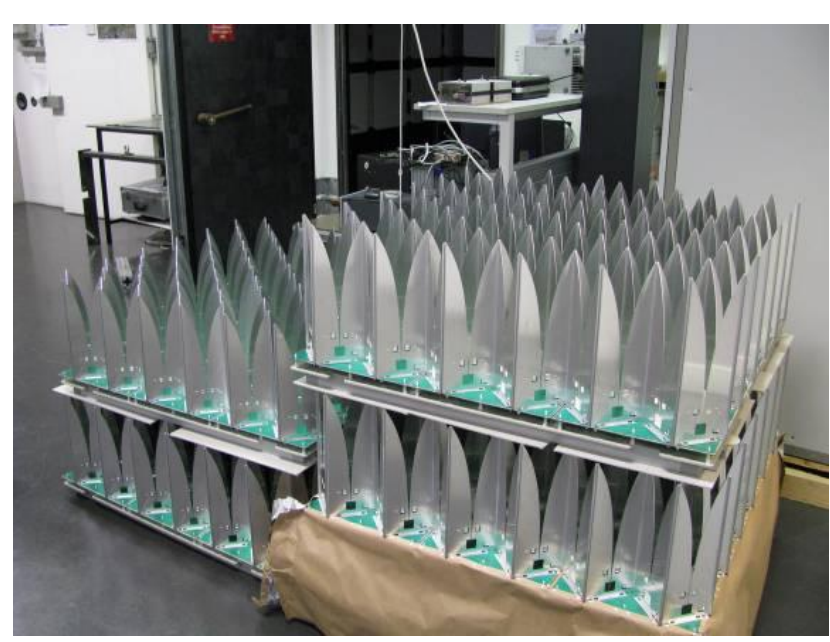

Fig. 4: EMBRACE modular tiles

Technology Readiness Levels. With LOFAR being installed and tested at this moment, TRL 9 system Test, Launch \& Operations was reached. The official opening of LOFAR will be in June 2010.

\section{Cost reduction by mass production processes}

With the SKADS demonstrator EMBRACE (Electronic MultiBeam Radio Astronomy ConcEpt) the numbers of identical parts are at the level that it can be used as an indicator for the full SKA numbers. The antenna element itself, of which 33000 were built, cost 1.5 Euro per piece. This includes material, cutting, forming integrated features, bending etcetera.

Special rods are used to connect the aluminum antenna elements. These rods were produced by extrusion. Extrusion is a nice process that can be applied for large quantities, but also is suitable for small series. This is because the mold is not that complicated and therefore not costly. The combination of the aluminum sheet antenna and the connecting aluminum extrusion element realizes a continual electrical connected antenna.

Another major step in cost reduction was accomplished by limiting the use of expensive PCB materials. A former ASTRON aperture array demonstrator did apply PCB material as antenna. In EMBRACE the PCB material was only applied at the feed line area where high accuracy and special RFproperties are required, what can be reached by etching process of RF-PCB materials. The aluminum radiator doesn't demand for such high accuracy and can be produced by laser cutting or, for larger amounts, by stamping.

Another way to reduce cost is the modular approach of the design. Separate assembled "blocks" were integrated and tested in a well conditioned environment. Testing and installing at the site can be done simultaneously. Fully functional and tested modules are delivered and malfunctioning parts are replaced at an early stage. Also the modularity makes it possible to start testing or even start observations with an unfinished array. The collecting area expands over time.

Also EMBRACE has a pretty high Technology Readiness Level. Not only the potential technology for a large instrument

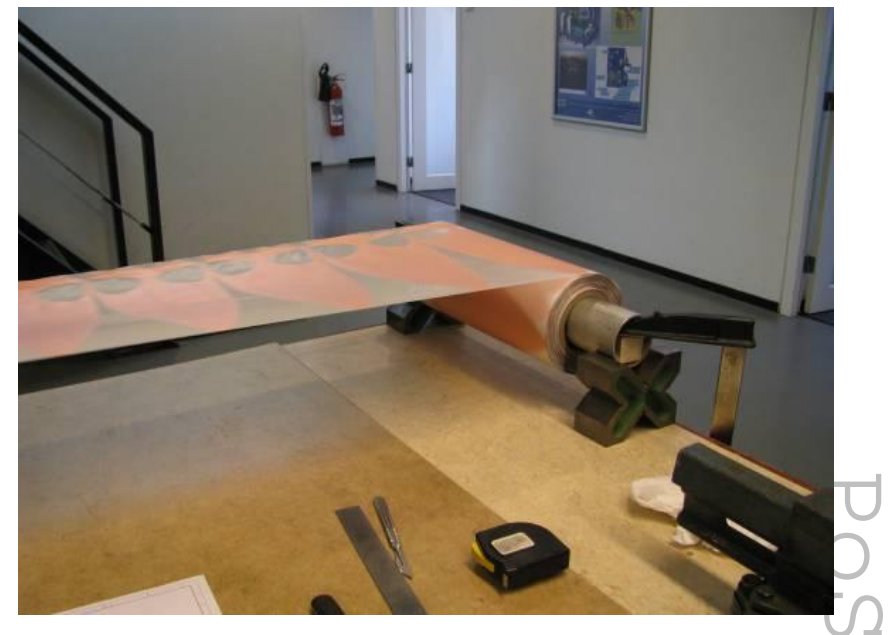

Fig. 5: Foil based vivaldi on roll

like SKA is demonstrated, but with its size EMBRACE also shows how to build a SKA subsystem / substation. This allows EMBRACE to be given TRL 6-7. A few steps need to be taken in order to reach the final level.

\section{Cost reduction by extreme low cost materials}

An example of how low cost materials can be used to build an advanced radio telescope can be found in FLOWPAD ${ }^{3}$, Foil based LOW cost PAcman Differential Dual polarized Demonstrator, which is part of the PACMAN project. The two low cost ingredients for this prototype are expanded polystyrene, known as packaging material, and thin foil produced on the roll. The use of the foil is interesting for several reasons. At first, by using a foil, less material is needed then when using a PCB, metal plate or sheet. Secondly it can be produced on the roll, making the required space for production and storage smaller, also handling is far easier. Third the process for making the antenna out of a foil is based on an additive production process, which basically means that only wanted cupper material on a layer will be added instead of removing of all the unwanted material of a cupper layer. So printing of cupper structure instead of the classical etching of cupper layer structure of the PCB process

The process used for producing this printed foil is known from the production of RFID tags. These tags can be found in several consumer products and are produced in large amounts for one time use only. For large quantities the cost for one foil based Vivaldi element is below one Euro. There are several possible foil materials useable for making the antenna foil. For the demonstrator a PET (polyester) foil was used. This is a relative low cost material also used for flower packaging. A thin silver seed layer in the form of conducting ink is printed onto the foil. A few microns are enough for electroplating and to grow a copper layer of 8 to 12 micron onto the seed layer.

When applying foil as vertical antenna, the flexible foil needs some kind of structure to keep it in its correct shape. Another solution is to apply a tension load on the foil to keep it mechanical stable. 
In the FLOWPAD ${ }^{3}$ design expanded polystyrene, EPS, is used as a supporting structure. This low loss material can be shaped for this purpose and the low density makes handling and assembly very easy. For the prototype the material was cut with a hot wire. This process is quite time consuming and relative expensive. For large numbers the structure part will be produced using a mould wherein the part will be formed. This already becomes interesting for medium series; the parts for the LOFAR HBA were made like this.

Another step for cost reduction within the foil based aperture array will be the integration of the LNA mounting. Within the antenna foil production process is it possible to mount a chip onto the foil. With the right design of chip size LNA the assembly of this chip can be integrated with the production of the foil. This will reduce assembly cost enormous. Also a cost reduction should be achieved by replacing the expensive RF connectors by integrating sensitive circuits on the foil. Examples of this are already shown in practice but these processes are not fully mature yet.

In spite of the fact that the process used to produce the antenna foils is a fully mature and frequently used production process the design itself hasn't reached the highest Technology Readiness Level yet. The principle and bits of the technology are demonstrated and show very good potential. The largest array built is $6,25 \mathrm{~m}^{2}$ of which only the center was active. This brings the FLOWPAD ${ }^{3}$ design to TRL 5-6. The demonstrated parts of the concepts are that promising that research to lift the concept up to the next TRL seems certainly worthwhile.

\section{Cost reduction by multi beaming}

A complete different approach to reduce cost is in the use of the instrument. With a FPA (Focal Plane Array) in the focus of a dish, multiple overlapping beams can be created. In this way the field of view is increased. Due to the increased field of view the observation speed is improved with a factor 20 .

Focal plane arrays can consist out of small series of parts, since over hundred identical components per FPA are needed. Within the APERTIF project (APERture Tile In Focus) the same production methods where used as within the EMBRACE project. Special extrusion rods were produced to keep the antennas in place. Obviously the strength and stiffness demands are higher since the front-end will move with the dish and the stability needs to be guaranteed. This makes the complexity of the instrument a bit higher, but the general approach is the same. Also laser cut aluminum vivaldi antennas were used, but the integrated features are a bit different. For the APERTIF prototype the design was made in such way that the element can be replaced separately. The electronics are supported by the vivaldi and each antenna can be removed by sliding them out of the array when needed.

With a few demonstrators and two systems already being installed in the WSRT, APERTIF has reached a high TRL as well. The technology used for the mechanical part of the frontend is known and comparable with EMBRACE. When the highest level is reached APERTIF will be ready to provide the complete WSRT with FPAs.

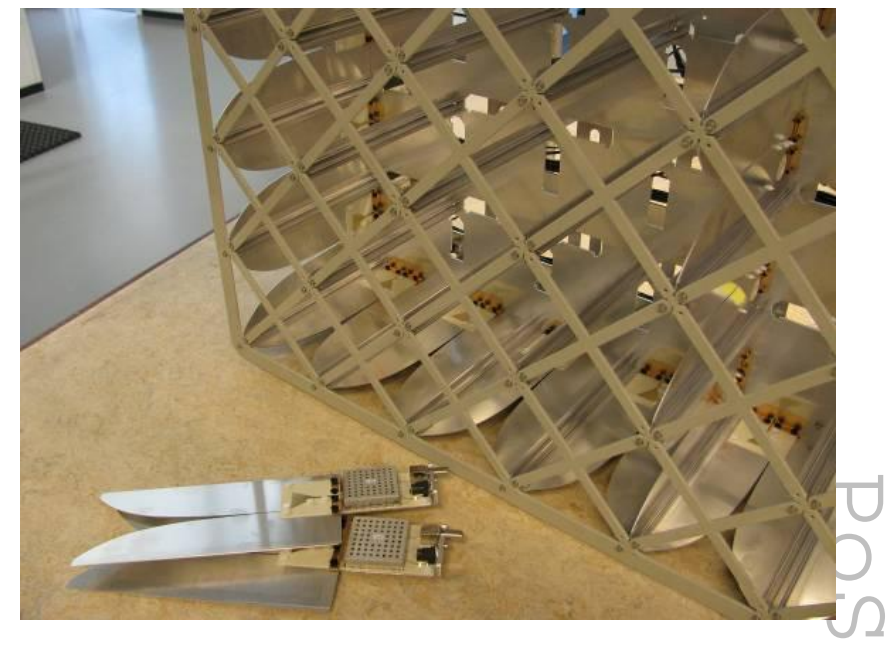

Fig. 6: APERTIF front-end

\section{Conclusions}

The demonstrators discussed in this paper all have different potentials for further development towards an SKA aperture array In general the cost of all demonstrators will decrease in future for the SKA production quantities. The larger quantities will lead to reduced investments per antenna since the cost of tools will be divided amongst more parts. Also production of larger quantities will justify to design and produce more specialized and integrated components and use more specialized production processes. Since there is a continuously development in the consumer market the SKA aperture array will automatically benefit from innovations, so that they can be used and bought as of the shelf mass market components.

Also on the electronic parts a higher level of integration (IC's) in combination with effect of Moore's law will decrease cost significantly.

shows the mechanical cost $/ \mathrm{m}^{2}$ of EMBRACE and FLOWPAD $^{3}$ as function of production numbers. It shows that the recurring cost like: material cost, production process time and assembly time are at larger quantities dominating the cost. It also shows that for FLOWPAD ${ }^{3}$ the nonrecurring costs are higher then EMBRACE. This is the result of for instance the expensive molds needed for the EPS molding process. At larger quantities however the short production time wins from the slower production processes used in EMBRACE. The lower cost of FLOWPAD ${ }^{3}$ at large quantities is realized by using: foil and foam based materials.

The cost $/ \mathrm{m}^{2}$ of an AA demonstrator is for a large part driven by the required area for that specific demonstrator. If only a few square meters is to be build, then a variety of mass production processes are not feasible due to their high the initial tooling cost. At +1000 square meter area more mass production processes become feasible.

\section{References}

M. Arts, M. Ivashina, O. Iupikov, L. Bakker and R. van den Brink. "Design Considerations of Tapered Slot Phased Array 


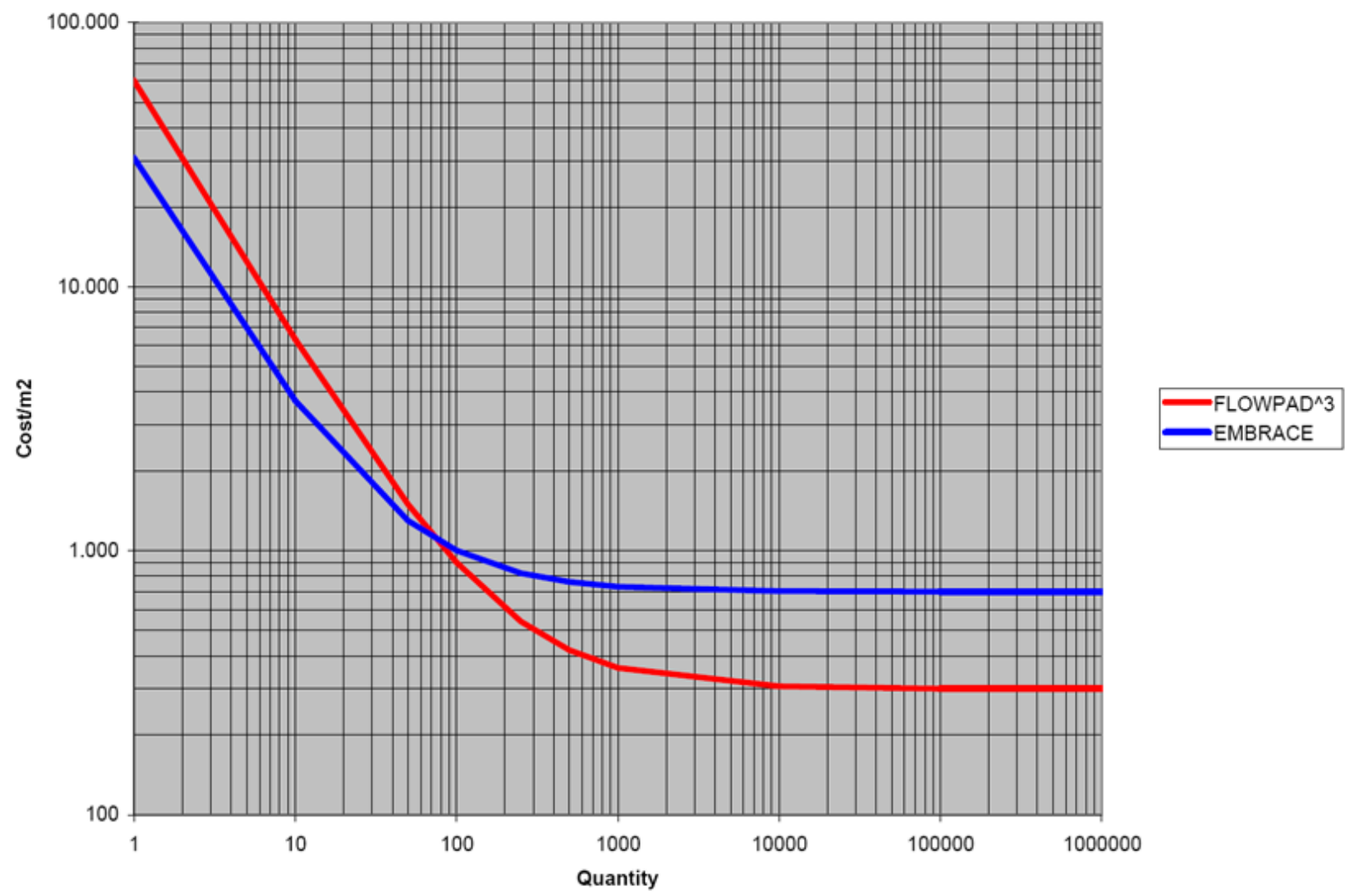

Fig. 7: Cost of frontend in relation to area (cost in Euros, quantity in square meter)

Feeds for Reflector Antennas" Accepted for: European Conference on Antennas and Propagation 2010, 12-16 April 2010, Barcelona.

J. Bregman, "Concept design for a Low Frequency Aray," Proc. SPIE conf. 4015, 2000.

H.R. Butcher, "LOFAR: First of a New Generation of Radio Telescopes", proceedings of the SPIE conf, 5489:537-544, October 2004.

A.W. Gunst, G.W. Kant, "Signal Transport and Processing at the LOFAR Remote Stations," URSI 2005, New Delhi, October, 23-29, 2005.

A.W. Gunst and M.J. Bentum "Signal processing aspects of the LOw Frequency ARray" 2007 IEEE International Conference on Signal Processing and Communications (ICSPC 2007), 24-27 November 2007, Dubai, United Arab Emirates

LOFAR Press release, First high quality wide field LOFAR image, Dwingeloo, the Netherlands, 25 April 2007

R.Maaskant, M.Popova, R.H. van den Brink "Towards the Design of a Low-Cost Wideband Demonstrator Tile for SKA" Proceedings of The European Conference on Antennas and Propagation: EuCAP 2006 (ESA SP- 626). 610 November 2006, Nice, France.

M.L. Philpott. personal communication Dept. of Mechanical \& Industrial Engineering, University of Illinois at UrbanaChampaign.
K. v.d. Schaaf, J.D. Bregman, C.M. de Vos, "Hybrid Cluster Computing Hardware and Software in the LOFAR Radio Telescope", proceedings of PDPTA 2003, CSREA Press.

C.M. de Vos, "LOFAR: The First of a New Generation of Radio Telescopes", Proceedings ICASSP, vol. 5, pp. 865868, March 2005.

M. A. W. Verheijen, T. A. Oosterloo, W. A. van Cappellen, L. Bakker,M. V. Ivashina and J. M. van der Hulst "APERTIF, a focal plane array for the WSRT",

S.J. Wijnholds, J.D. Bregman, A. Boonstra, "Sky Noise Limited Snapshot Imaging in the Presence of RFI with LOFAR's Initial Test Station," Experimental Astronomy, V17, pp35-42, 2004 